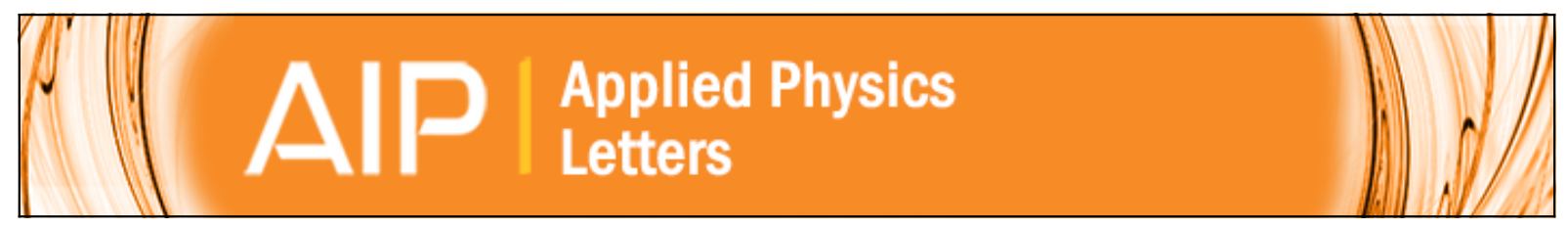

\title{
Analysis of deep levels in a phenylenevinylene polymer by transient capacitance methods
}

H. L. Gomes, P. Stallinga, H. Rost, A. B. Holmes, M. G. Harrison, and R. H. Friend

Citation: Applied Physics Letters 74, 1144 (1999); doi: 10.1063/1.123469

View online: http://dx.doi.org/10.1063/1.123469

View Table of Contents: http://scitation.aip.org/content/aip/journal/apl/74/8?ver=pdfcov

Published by the AIP Publishing

\section{Articles you may be interested in}

Deep levels in as-grown and electron-irradiated n-type GaN studied by deep level transient spectroscopy and minority carrier transient spectroscopy

J. Appl. Phys. 119, 095707 (2016); 10.1063/1.4943029

Observed trapping of minority-carrier electrons in $p$-type GaAsN during deep-level transient spectroscopy measurement

Appl. Phys. Lett. 86, 072109 (2005); 10.1063/1.1865328

Deep levels created by low energy electron irradiation in $4 \mathrm{H}$ - SiC

J. Appl. Phys. 96, 4909 (2004); 10.1063/1.1778819

Determination of traps in poly(p-phenylene vinylene) light emitting diodes by charge-based deep level transient spectroscopy

J. Appl. Phys. 90, 4196 (2001); 10.1063/1.1403669

Deep levels in Er-doped liquid phase epitaxy grown silicon

Appl. Phys. Lett. 72, 468 (1998); 10.1063/1.120788

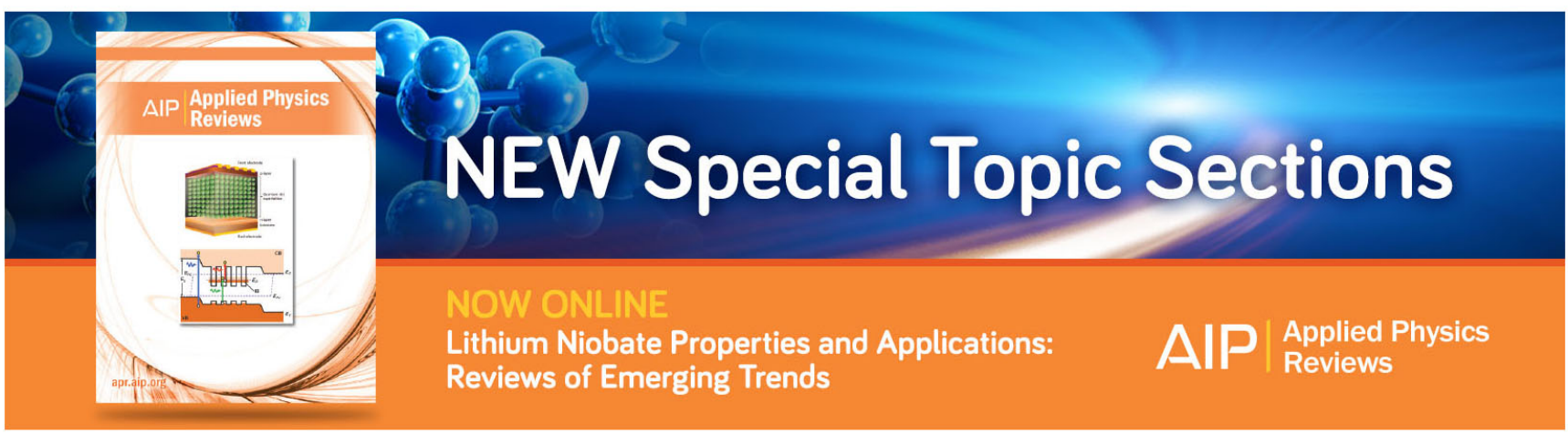




\title{
Analysis of deep levels in a phenylenevinylene polymer by transient capacitance methods
}

\author{
H. L. Gomes and P. Stallinga \\ Universidade do Algarve, UCEH, Campus de Gambelas, 8000 Faro, Portugal \\ H. Rost and A. B. Holmes \\ Melville Laboratory for Polymer Synthesis, Cambridge CB2 3RA, United Kingdom \\ M. G. Harrison and R. H. Friend \\ Cavendish Laboratory, Cambridge CB3 OHE, United Kingdom
}

(Received 17 November 1998; accepted for publication 16 December 1998)

\begin{abstract}
Transient capacitance methods were applied to the depletion region of an abrupt asymmetric $n^{+}$ $-p$ junction of silicon and unintentionally doped poly[2-methoxy, 5 ethyl (2' hexyloxy) paraphenylenevinylene] (MEH-PPV). Studies in the temperature range 100-300 K show the presence of a majority-carrier trap at $1.0 \mathrm{eV}$ and two minority traps at 0.7 and $1.3 \mathrm{eV}$, respectively. There is an indication for more levels for which the activation energy could not be determined. Furthermore, admittance data reveal a bulk activation energy for conduction of $0.12 \mathrm{eV}$, suggesting the presence of an additional shallow acceptor state. () 1999 American Institute of Physics. [S0003-6951(99)02308-6]
\end{abstract}

Poly[2-methoxy, 5 ethyl (2' hexyloxy) paraphenylenevinylene] (MEH-PPV) is an electroluminescent conjugated polymer now being developed as a material for use in display technology. ${ }^{1-5}$ It is well known that the luminescence is strongly affected by carrier traps which exist at energies within the band gap, and which may act as nonradiative carrier recombination centers, thus reducing the light emission efficiency. Time-of-flight measurements ${ }^{6}$ have shown that electrons, in contrast to holes, are strongly trapped in PPV. The resulting unbalanced electron and hole concentrations will have deleterious effects on luminescence efficiency. In addition, these deep levels may play an important role in the conduction mechanism.

Thermally stimulated currents in ITO/PPV/Al devices ${ }^{7,8}$ indicate the presence of several distinct gap states with energies in the range $0.05-0.2$ and $0.6-1.0 \mathrm{eV}$. It was also reported $^{9}$ that the current-voltage characteristics of ITO/ PPV/Al devices can be fitted using an exponential distribution of traps with a characteristic energy of $0.15 \mathrm{eV}$. Deep traps have been assigned to the influence of the environment, while the shallow ones are assigned to doping by the ITO. ${ }^{8}$ However, it is clear that the microscopic nature of these trap states and their role in the charge transport need further investigation.

Although capacitance transient techniques, such as deep level transient spectroscopy (DLTS), have been acknowledged as one of the most powerful methods to detect and characterize electrically active defects in semiconductors, only a few studies ${ }^{10}$ have used these techniques to characterize semiconducting conjugated polymers. The work here represents the first use of capacitance transients to observe the thermal emission processes due to trapped carriers in $\mathrm{MEH}$ PPV. In order to prime both minority and majority-carrier traps we used a $p$ - $n$ junction. Moreover, an abrupt asymmetric $n^{+}$( silicon) $-p$ (MEH-PPV) junction is preferred because the depletion layer is then mostly in the polymer side. Con- sequently, the depletion capacitance will be more sensitive to traps in the lightly doped material, the MEH-PPV.

Thin films of MEH-PPV (0.1-0.2 $\mu \mathrm{m}$ thick) were deposited by spin coating onto $n^{+}$-silicon substrates. Before deposition the silicon substrates were cleaned with diluted $\mathrm{HF}$ to remove any residual oxide layer and were rinsed several times in ultrapure water. After spin coating the films were immediately loaded into a turbopumped evaporator and gold was evaporated through a perforated mask to form an array of circular electrodes ( $2 \mathrm{~mm}$ in diameter). The devices were mounted in a temperature controlled sample holder located inside a steel chamber evacuated to less than $10^{-5}$ mbar. Small-signal admittance measurements over the range $50 \mathrm{~Hz}$ to $1 \mathrm{MHz}$ were carried out with a Fluke PM 6306 RCL meter. The $I-V$ curves were recorded with a Keithley 487 Picoammeter/voltage source. The sample temperature was varied in the range $100-300 \mathrm{~K}$ and measured with a chromel-alumel thermocouple placed on the substrate close to the devices. All the measurements were carried out in the dark. Despite all the precautions to minimize air exposure, it cannot be excluded that some residual oxygen was introduced during the spin coating deposition. This oxygen could have slowly diffused towards the silicon surface and created a very thin oxide layer. Such a layer offers little resistance to the passage of current, and the device may resemble a tunnel metal-insulator-semiconductor (MIS) diode. ${ }^{11}$

The devices exhibit good diode behavior, with a typical current rectification ratio higher than $10^{3}$ at a bias of $|0.5 \mathrm{~V}|$ and an ideality factor of 1.6. Figure 1 shows the roomtemperature current-voltage characteristics of a typical device. Figure 2 shows the loss $(G / \omega)$ and the capacitance $(C)$ versus frequency at room temperature and at $116 \mathrm{~K}$. The prominent loss peak and capacitance step-at $500 \mathrm{kHz}$ at room temperature-is related with the $R C$ time constant of the diode. This relaxation frequency decreases to below 1 $\mathrm{kHz}$ only when the device is cooled to the lowest tempera- 


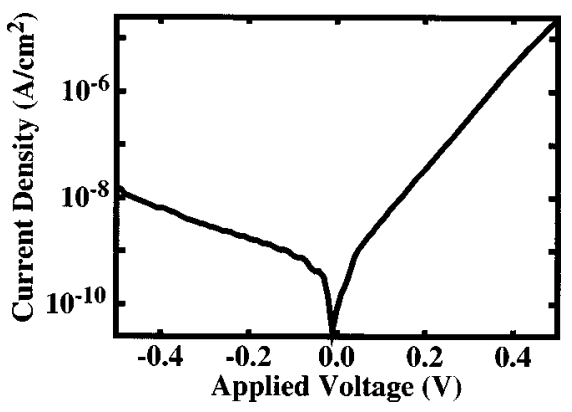

FIG. 1. $I-V$ curve at room temperature showing the high rectification ratio (more than 1000) and ideality factor of 1.6.

tures. Therefore, using a low frequency ac voltage at $1 \mathrm{kHz}$, we can measure capacitance transients over a large temperature range, without limitations due to the diode time constant. The capacitance and conductance data plotted as a loss tangent ( $\tan \delta=G / \omega C$ ) will show a peak at a particular frequency $\left(\nu_{\max }\right)$. It has been shown ${ }^{12}$ that, assuming the depletion layer resistance is much higher than the bulk resistance, the temperature dependence of $\nu_{\text {max }}$ should follow the same temperature dependence as the conductance of the bulk material. We can extract therefore the polymer bulk activation energy from the slope of a plot of $\ln \left(\nu_{\max }\right)$ vs $1 / T$. In our case $E_{a}$ is $0.12 \mathrm{eV}$ [Fig. 2(b)]. This may correspond to the shallowest ionized acceptor level responsible for conduction. From a plot of reciprocal square capacitance versus applied voltage, we estimated an ionized acceptor doping concentration of the order of $10^{16} \mathrm{~cm}^{-3}$.

In obtaining trap levels from capacitance transients, it is important to pay attention to exponentiality since nonexponential decays can complicate the data analysis. The capacitance transients recorded usually show an immediate steplike response to the voltage change followed by a long-lived signal composed of, in our case, a small number of simple exponentials. The sign of the component depends on the character of the trap. For a majority trap, we expect a negative capacitance transient (upward trend), whereas the transient due to minority carrier trap is positive (downward trend). ${ }^{13}$

Figure 3 shows that, by an appropriate pulse scheme, it is possible to prime either the minority- or the majoritycarrier trap levels. The majority carrier traps can be detected using a filling pulse of zero bias, and minority-carrier traps are revealed by injecting electrons into the polymer in a forward bias $(1.0 \mathrm{~V})$ pulse. A complication is that forward-
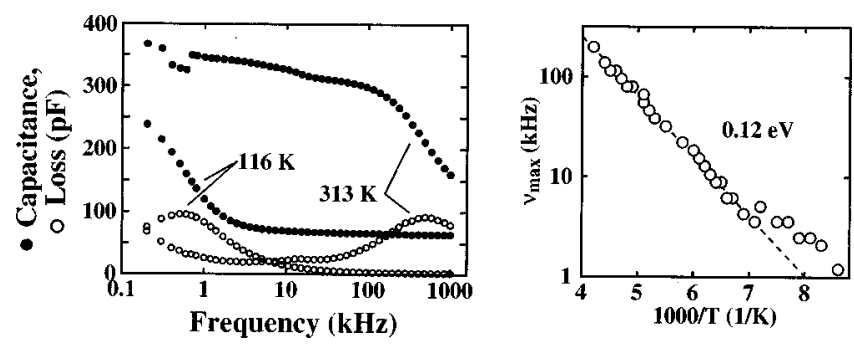

FIG. 2. (a) Capacitance ( $C$; solid circles) and loss $(1 / \omega R$; open circles) for the two temperatures 313 and $116 \mathrm{~K}$. The cut-off frequency of the diode moves from 500 to below $1 \mathrm{kHz}$. (b) Shows the position of the peak in the loss tangent ( $\tan \delta=1 / \omega R C$ ) as a function of temperature. This reveals the bulk activation energy of $0.12 \mathrm{eV}$.

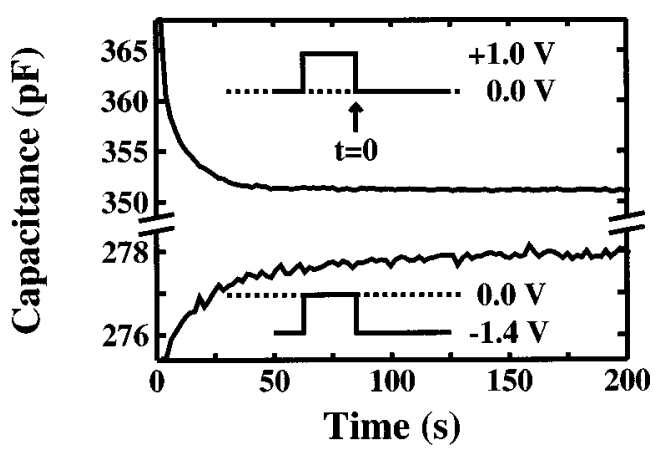

FIG. 3. By an appropriate bias pulse, the transient can be made to show a minority trap (top) recognizable by the downward trend, or majority trap (bottom) in a upward trend.

injecting bias pulses, because of the collapsing of the depletion range, can also significantly perturb the occupation of interface states (and give nonexponential contributions to the capacitance transients), which is not desirable because our interest is to prime only bulk traps. Therefore, during the experiments the sample was kept under zero bias and then subjected to a reverse step of $-0.8 \mathrm{~V}$. The corresponding transient capacitance under reverse bias was recorded at a series of stabilized temperatures to provide the time constants $(\tau)$.

Although we did not forward bias the $p$ - $n$ junction, we observe over certain temperature ranges a reversal in the sign of the capacitance change corresponding to a change from a majority-type behavior to a minority-type behavior. This reversal in the sign of capacitance change is illustrated in Fig. 4 , where for a certain temperature both types of behavior are visible at the same time. The observation of minority-type transients suggests that electron diffusion from the $n^{+}$silicon is enough to fill some minority traps in the polymer.

The activation energy of the trap is calculated by noting that the emission rate, $e_{n}=1 / \tau$, of the carriers from a trap of depth $E_{a}$ at temperature $T$ is given by Eq. (1) where $k$ is the Boltzmann constant. ${ }^{13}$

$$
e_{n}=1 / \tau \propto T^{2} \exp \left(-E_{a} / k T\right) .
$$

The equation was applied to the data shown in Fig. 5. The solid and open circles represent majority and minority traps, respectively. There is a clear evidence for at least three trap levels, two minority ones (activation energies: 0.7 and $1.3 \mathrm{eV})$ and a majority one $\left(E_{a}=1.0 \mathrm{eV}\right)$. The data points related to these traps are so closely spaced, that the exact

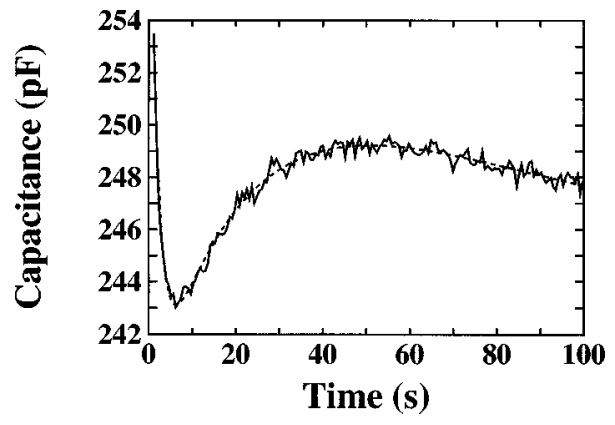

FIG. 4. When the emission rates of two traps are close enough together, transients can even have both types of behavior shown in Fig. 3 visible at the same time. The above picture reveals two minority traps and in between a majority trap. 


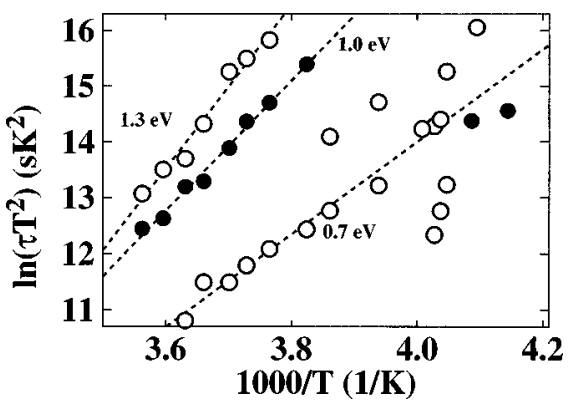

FIG. 5. Summary of the measurements. The corrected characteristic time of the transients $\tau T^{2}$ are plotted as a function of reciprocal temperature (1/T) to reveal the activation energies of the associated traps. Open circles represent minority traps while full circles correspond to majority traps.

determination of the time constants is cumbersome and depends for instance on the fitting procedure. Hence, the activation energy of the trap levels have a wide margin of error (about $0.2 \mathrm{eV}$ ). It has to be noted, however, that there is no doubt of the existence of the above mentioned levels.

In summary, transient capacitance spectroscopy techniques were applied to characterize carrier traps in unintentionally doped MEH-PPV. Our results show that, despite the chemical differences between inorganic semiconductors and conjugated polymers, the original junction DLTS technique proposed by Lang ${ }^{13}$ can be applied to these new materials. Furthermore, the use of a $p-n$ junction enabled us to observe minority traps for the first time in MEH-PPV. Using this technique, a majority trap and two minority-carrier traps were found. The found activation energies agree with the values $(0.6-1.0 \mathrm{eV})$ reported by others using thermally stimulated currents in PPV prepared by the precursor route. ${ }^{7,8}$ The results provide evidence for more levels for which the activation energies could not be determined. Subsidiary experiments using admittance measurements gave a bulk activation energy for conduction of $0.12 \mathrm{eV}$, suggesting the presence of a shallow acceptor state.

The authors are grateful to Professor D. M. Taylor for valuable discussions. They are pleased also to acknowledge the financial support of the TMR program, SELOA, Contract No. ERBFMRX-CT96-0083.

${ }^{1}$ J. H. Burroughes, D. D. C. Bradley, A. R. Brown, R. N. Marks, K. Mackay, R. H. Friend, P. L. Burn, and A. B. Holmes, Nature (London) 347, 539 (1990).

${ }^{2}$ D. Braun and A. J. Heeger, Appl. Phys. Lett. 58, 1982 (1991).

${ }^{3}$ W. Rieß, Organic Electroluminescent Materials and Devices (Gordon and Breach, New York, 1996).

${ }^{4}$ N. Tesler, N. T. Harrison, and R. H. Friend, Adv. Mater. 10, 64 (1988).

${ }^{5}$ A. Kraft, A. C. Grimsdale, and A. B. Holmes, Angew. Chem. Int. Ed. Engl. 37, 402 (1988).

${ }^{6}$ H. Antoniades, M. A. Abkowitz, and B. R. Hsieh, Appl. Phys. Lett. 65, 2030 (1994).

${ }^{7}$ J. Scherbel, P. H. Nguyen, G. Paasch, W. Brütting, and M. Schwoerer, J. Appl. Phys. 83, 5045 (1988).

${ }^{8}$ W. Brütting, S. Karg, M. Meier, E. Werner, M. Herold, and M. Schwoerer, Proc. ECME'97, Cambridge, 1997.

${ }^{9}$ A. J. Campbell, D. D. C. Bradley, and D. G. Lidzey, J. Appl. Phys. 82, 6326 (1997).

${ }^{10}$ G. W. Jones, D. M. Taylor, and H. L. Gomes, Synth. Met. 85, 1342 (1997).

${ }^{11}$ M. A. Green and J. Schewchun, J. Appl. Phys. 46, 5185 (1975).

${ }^{12}$ D. M. Taylor and H. L. Gomes, J. Phys. D 28, 2554 (1995).

${ }^{13}$ D. V. Lang, J. Appl. Phys. 45, 3023 (1974). 